\title{
A fast feedback controlled magnetic drive for the ASDEX Upgrade fast-ion loss detectors
}

\author{
J. Ayllon-Guerola, ${ }^{1,2, a)}$ J. Gonzalez-Martin, ${ }^{1,2}$ M. Garcia-Munoz, ${ }^{1,2}$ J. Rivero-Rodriguez, ${ }^{1,2}$ \\ A. Herrmann, ${ }^{3}$ S. Vorbrugg, ${ }^{3}$ P. Leitenstern, ${ }^{3}$ S. Zoletnik, ${ }^{4}$ J. Galdon, ${ }^{1,2}$ J. Garcia Lopez,,${ }^{1,2}$ \\ M. Rodriguez-Ramos, ${ }^{1,2}$ L. Sanchis-Sanchez, ${ }^{1,2}$ A. D. Dominguez, ${ }^{1,2}$ M. Kocan, ${ }^{5}$ \\ J. P. Gunn, ${ }^{6}$ D. Garcia-Vallejo, ${ }^{7}$ and J. Dominguez ${ }^{7}$ \\ ${ }^{1}$ Department of Atomic, Molecular and Nuclear Physics, Faculty of Physics, University of Seville, \\ 41012 Seville, Spain \\ ${ }^{2}$ CNA (U. Sevilla, CSIC, J. de Andalucia), Sevilla, Spain \\ ${ }^{3}$ Max-Planck-Institut für Plasmaphysik, Garching, Germany \\ ${ }^{4}$ Wigner RCP, Budapest, Hungary \\ ${ }^{5}$ ITER Organization, Route de Vinon sur Verdon, 13115 St Paul Lez Durance, France \\ ${ }^{6}$ CEA, IRFM, F-13108 Saint Paul Lez Durance, France \\ ${ }^{7}$ ETSI, University of Seville, 41092 Seville, Spain
}

(Presented 9 June 2016; received 6 June 2016; accepted 28 June 2016; published online 3 August 2016)

\begin{abstract}
A magnetically driven fast-ion loss detector system for the ASDEX Upgrade tokamak has been designed and will be presented here. The device is feedback controlled to adapt the detector head position to the heat load and physics requirements. Dynamic simulations have been performed taking into account effects such as friction, coil self-induction, and eddy currents. A real time positioning control algorithm to maximize the detector operational window has been developed. This algorithm considers dynamical behavior and mechanical resistance as well as measured and predicted thermal loads. The mechanical design and real time predictive algorithm presented here may be used for other reciprocating systems. [http://dx.doi.org/10.1063/1.4959913]
\end{abstract}

\section{BACKGROUND}

Scintillator based fast-ion loss detectors (FILDs) are installed in virtually all major tokamaks and stellarators to study the fast-ion losses induced by magnetohydrodynamic (MHD) fluctuations. ${ }^{1-3}$ FILD systems provide velocity-space measurements of fast-ion losses with the Alfvenic temporal resolution. This information is crucial to identify the MHD fluctuations responsible for the actual fast-ion losses and to understand the wave-particle interaction underlying the transport mechanism. As charged particle collectors, FILD systems must work a few gyroradii away from the separatrix and are, therefore, exposed to high heat load that limits significantly the duration of the measurements.

The FILDs installed in ASDEX Upgrade (AUG) are designed to operate in a fixed position during plasma discharges. The insertion length must be predefined and, when the discharge is over, they are automatically retracted. To protect both tokamak and device integrity, a safety protocol allows aborting the discharge if high heat loads are measured. Providing the detector with a temperature (or any other thermal load measure) feedback control will allow it to automatically retract if needed, e.g., if heat loads are suddenly increased due to plasma displacements and MHD events. Given the temporal scale associated to thermal variations close to the plasma, fast displacements will be required to, in a very short time, send the

Note: Contributed paper, published as part of the Proceedings of the 21st Topical Conference on High-Temperature Plasma Diagnostics, Madison, Wisconsin, USA, June 2016.

a) Author to whom correspondence should be addressed. Electronic mail: jayllon@us.es. probe back to a safe position. Magnetically driven actuators are a good choice to produce fast movements in a robust and reliable way. ${ }^{4-6}$ In this type of drive, an energized coil takes advantage of the magnetic field existing in the tokamak and rotates to align its axis with the field lines. So, this rotation is transmitted to the probe head.

Magnetic drives used at AUG to expose probes are designed to move between two fixed positions ${ }^{4}$ and, when the input voltage is zero, are retracted by a spring. Devices installed in other tokamaks ${ }^{5}$ have been used to bring probes close to the plasma, by a continuous insertion-retraction movement without holding it in the inserted position. This can be done by changing the input voltage polarity before the probe reaches its inner limiter. However, FILDs must stay in measurement position close to the plasma for as much time as possible. The measurement cycle is given by a compromise between measurement requirements and heat load. This balance will define the time the probe can stay in measurement position and the reciprocating timing.

In Section II, the design of the magnetically driven FILD and its multibody model are presented. Next, in Section III, dynamic simulations are described and the detector structural integrity is assessed. Finally, in Section IV, an algorithm for real time positioning with heat load interlock is presented.

\section{FILD MECHANICAL DESIGN, MULTIBODY MODEL, AND COIL DIMENSIONING}

The detector mechanical design is shown in Fig. 1(a). The probe head moves following a straight line, guided by two sets of four ball bearings each. The coil torque 

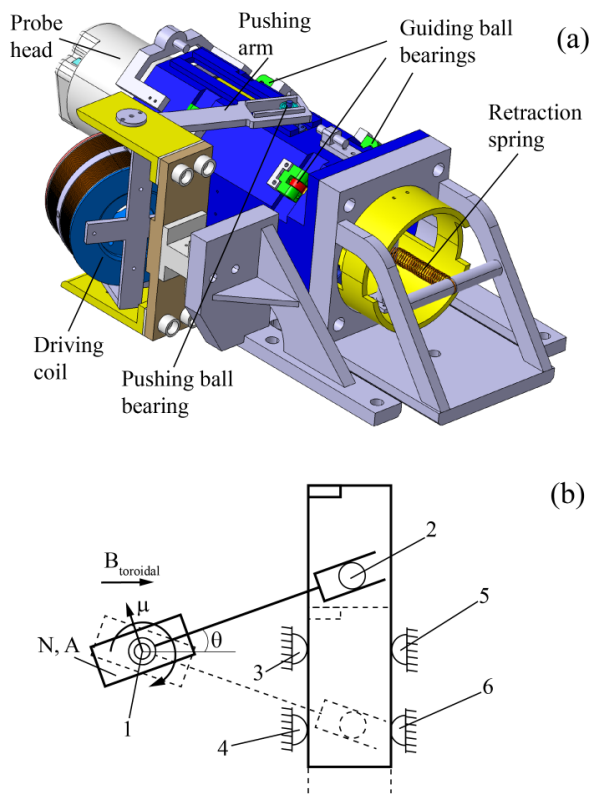

(b)

FIG. 1. (a) Magnetically driven FILD CAD design. (b) 2D multibody model used in dynamic simulations: A revolute joint (1) has been defined in the coil rotation axis. A circumference-line-type cam joint (2) models the contact between the pushing arm and the bearing. The contact between the probe head holder and each guiding bearing (3), (4), (5), and (6) has been defined as point-line-type cam joint.

is transmitted by a double arm, where a couple of ball bearings are allowed to move along respective grooves. A resistive transducer is installed to monitor the probe position and a linear spring is provided to hold the device in parking position when not being used. A 2D multibody numerical model is illustrated in Fig. 1(b), where kinematical joints have been introduced to properly account for the different elements connections and to include effects derived from friction. This model will be used in Section III to investigate the detector dynamic behavior.

When a voltage $V$ is applied to the coil, a torque $\tau_{\text {coil }}$ $=\mu B \cos \theta$ forces it to align its magnetic moment $\mu=N I A$ with the total magnetic field $B$, as shown schematically in Fig. 1(b). The number of windings $N$ of the coil and the cross section of each winding $A$ (fixed by constructive constraints) need to be determined to assure that the detector motion fulfills the requirements. Once a $V$ is applied to the coil, and it starts rotating, the current $I$ in the drive circuit can be expressed as

$$
I=\frac{1}{R}\left(V-N B A \cos (\theta) \dot{\theta}-L_{\text {self }} \dot{I}\right),
$$

where $\theta$ is the angle rotated by the coil. The negative terms in the parentheses correspond to the back electromotive force and coil self-inductance $L_{\text {self }}$, and develop the torques $\tau_{e m f}$ and $\tau_{\text {self }}$, respectively. Then, the torque produced by the coil, $\tau_{\text {coil }}$, can be considered as the sum of three effects,

$$
\tau_{c o i l}=\tau_{\text {appl }}+\tau_{\text {emf }}+\tau_{\text {self }},
$$

the last two of them countering the effect of the applied $V$ and damping the drive motion. Therefore, $\tau_{\text {coil }}$ needs to overcome the opposite torques produced by the spring $\tau_{\text {spring, }}$, the probe inertia $\tau_{\text {probe, }}$, and the eddy currents in moving parts $\tau_{\text {eddy }}$, and provide an angular acceleration $\ddot{\theta}$ to the coil according to the equation

$$
i \ddot{\theta}=\tau_{\text {coil }}+\tau_{\text {eddy }}+\tau_{\text {spring }}+\tau_{\text {probe }},
$$

where $i=4 \times 10^{-3} \mathrm{~kg} \mathrm{~m}^{2}$ is the moment of inertia of the coil plus its supporting arm.

Following the analysis presented in Ref. 4, the optimal coil parameters have been obtained. To that end, the torque equilibrium equation (Eq. (3)), once conveniently linearized, has been used to properly estimate the number of windings $N$ and the voltage $V$ needed to (1) hold the probe beyond the inserted position overcoming the spring force and (2) operate the detector in overdamped regime. Additional constraints have been set to provide an approximated minimum retraction time around $50 \mathrm{~ms}$ and to limit the applied voltage to a maximum value of $20 \mathrm{~V}$. The optimal coil parameters obtained are $\mathrm{N}=160$ and $\mathrm{A}=0.006 \mathrm{~m}^{2}$.

\section{DYNAMIC SIMULATIONS AND STRUCTURAL INTEGRITY}

The detector dynamic behavior has been characterized, using the multibody model (see Fig. 1(b)), by solving the Newton-Euler differential algebraic equations ${ }^{7}$

$$
\begin{gathered}
M(q) \ddot{q}=Q(q, \dot{q}, \lambda, I, t), \\
C(q)=0, \\
\dot{I}=h(q, \dot{q}, I, V),
\end{gathered}
$$

where $q$ is the generalized coordinates vector, $M$ the generalized mass matrix, $Q$ the generalized external forces vector, and $C$ the geometric constraints vector. To take into account the friction effect, the reaction forces in the joints have been evaluated through Lagrange multipliers, $\lambda$. The associated friction forces have been updated within each integration step.

Fig. 2(a) shows the simulated measurement cycle, where the probe head position is represented for two friction coefficient values $(\mu=0$ and $\mu=0.6)$. Four phases can be identified in the simulated measurement cycle: (1) an initial one where a constant voltage (open loop) is applied until the probe head reaches a pre-defined insertion depth. No fast insertion is needed since the device takes advantage of the magnetic field existing in the tokamak a few seconds before the plasma discharge starts. Then, (2) the position is controlled by a proportional integral derivative (PID) controller which compares the probe head target and current positions and adjusts the voltage delivered to the coil to smoothly reach the measurement stroke $(80 \mathrm{~mm})$. The position is held until the retraction phase starts (3) and a reversed polarity open loop voltage (retraction voltage $V_{\text {ret }}=10 \mathrm{~V}$ ) is applied to fast retract the probe to a safe position in the limiter shadow, at a distance of $40 \mathrm{~mm}$ from the parking position. The retraction time depends on the friction coefficient (as shown in Fig. 2(b)) and can be adjusted by modifying $V_{\text {ret }}$. Finally (4) the detector is 

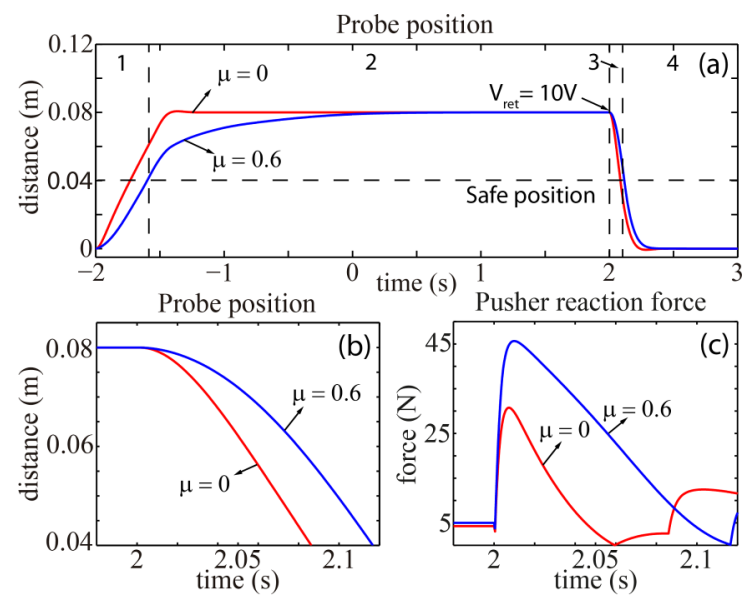

FIG. 2. (a) Probe head position as a function of time. Different values for friction coefficient ( $\mu=0$ and $\mu=0.6$ ) have been considered. Different retraction times (b) and reaction forces (c) in the pushing bearing (see Fig. 1) depending on the friction coefficient are shown during the retraction phase for $V_{\text {ret }}=10 \mathrm{~V}$.

fully retracted to its parking position controlled by the PID.

The maximum reaction force in the mechanism, represented in Fig. 2(c), occurs in the pushing bearing joint (see Fig. 1(b)) during the retraction phase. By evaluating the mechanical stresses induced by the reaction forces, conducting finite element (FE) analysis, it has been concluded that the pushing bearing rod is the weakest structural element in the detector, since it is subjected to the highest mechanical stress $\sigma_{\max }$. If this stress is compared to a material limit value (e.g., the yielding limit $\sigma_{y}$ ) a structural safety factor for the detector, $S F_{S}$, can be defined as

$$
\mathrm{SF}_{\mathrm{S}}=\sigma_{\mathrm{y}} / \sigma_{\max } .
$$

In Fig. 3, the detector safety factor is represented as a function of the retraction time, the friction coefficient, and $V_{r e t}$. When $S F_{S}$ equals the value 1 or lower, local plasticity occurs in the pushing bearing rod. It is important to avoid permanent deformations in the system because this

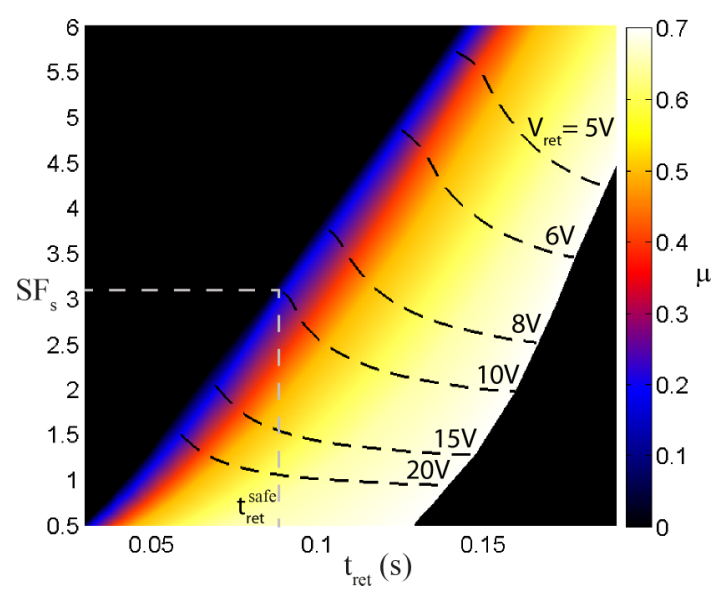

FIG. 3. Structural safety factor of the FILD system as a function of the retraction time, the friction coefficient, and the voltage applied during the retraction phase. A system with $\mu=0$ and $V_{\text {ret }}=10 \mathrm{~V}$ will retract in $t_{\text {ret }}^{\text {safe }}$ $=0.08 \mathrm{~s}$ with a structural safety factor higher than 3 .
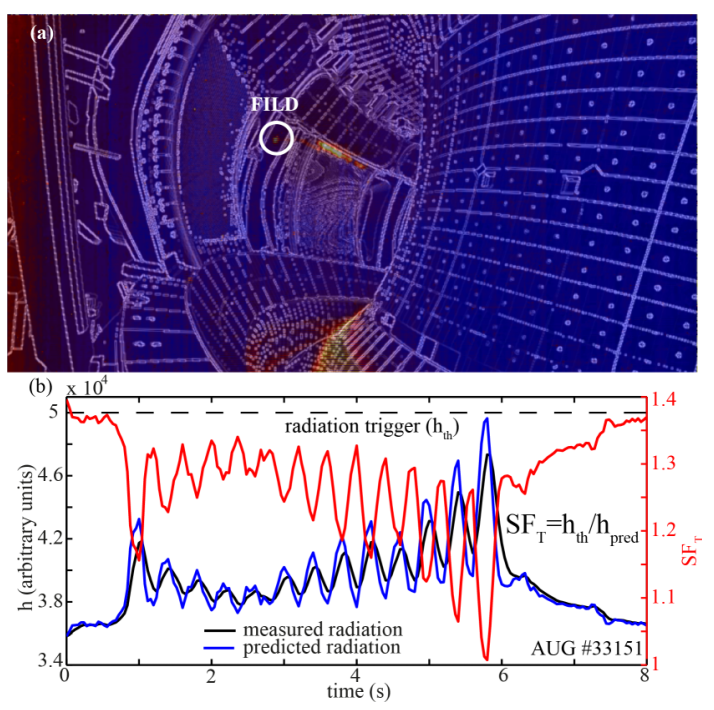

FIG. 4. (a) A camera monitors the radiation emitted by the detector in the visible range during the plasma discharge, which is related to its temperature. The control algorithm (b) allows determining a thermal safety factor $S F_{T}$ using a trigger value as a reference.

can lead to poor performance in the guiding system. This structural safety factor can be used to define a minimum safe retraction time $t_{\text {ret }}^{\text {safe }}$ which is a constraint parameter in the thermal-safe operation algorithm, as will be explained in Section IV.

\section{FEEDBACK CONTROL FOR REAL TIME POSITIONING}

The goal of the real time feedback control system is to keep the detector in measurement position as long as possible without comprising its integrity due to both high stresses and/or thermal loads. Additionally, if overheating is detected, the device needs to retract before reaching the general AUG thermal threshold, i.e., before the plasma discharge is aborted.

If the radiation emitted by the probe head is monitored during the detector operation, $t_{\text {ret }}^{\text {safe }}$ (see Fig. 3) can be used to obtain a thermal safety factor $S F_{T}$, shown in Fig. 4(b) as a function of time. Using a conservative approach, it can be assumed that, at any given time $t_{i}$, the device retracts within $t_{r e t}^{\text {safe }}$, while thermal load still grows linearly maintaining the slope calculated at $t_{i}$. During this retraction time the emitted radiation will reach a predicted value $h_{\text {pred }}$, which can be compared to a threshold value $h_{t h}$, lower than the general AUG threshold. When $h_{\text {pred }}$ equals $h_{t h}, S F_{T}$ will be equal to 1 and the detector will be retracted.

\section{SUMMARY}

A conceptual design for a magnetically driven FILD has been developed for the AUG tokamak. Dynamic and FE simulations allowed defining a structural safety factor depending on the retraction time and friction coefficient. A thermal-safe operation algorithm is proposed to optimize the detector operational window in a wide variety of plasma scenarios. 


\section{ACKNOWLEDGMENTS}

The authors would like to thank the ASDEX Upgrade Team and especially W. Zeidner, T. Vierle, and W. Popken for their help with the technical aspects of this project, also E. Freire for his advice regarding the multibody model numerical solution.

This work has been carried out within the framework of the EUROfusion Consortium and has received funding from the Euratom research and training programme 2014-
2018 under Grant Agreement No. 633053. The views and opinions expressed herein do not necessarily reflect those of the European Commission.

${ }^{1}$ M. Garcia-Munoz et al., Phys. Rev. Lett. 104, 185002 (2010).

${ }^{2}$ D. S. Darrow, Rev. Sci. Instrum. 79, 023502 (2008).

${ }^{3}$ R. K. Fisher et al., Rev. Sci. Instrum. 81, 10D307 (2010).

${ }^{4}$ A. Schmid, A. Hermann, V. Rohde, M. Maraschek, H. W. Müller, and ASDEX Upgrade Team, Rev. Sci. Instrum. 78, 053502 (2007).

${ }^{5}$ J. P. Gunn and J.-Y. Pascal, Rev. Sci. Instrum. 82, 123505 (2011).

${ }^{6}$ N. Smick and B. LaBombard, Rev. Sci. Instrum. 80, 023502 (2009).

${ }^{7}$ W. Schiehlen, Multibody Syst. Dyn. 1, 149 (1997). 